\title{
Correction to: MultiMedia Modeling
}

Yong Man Ro, Wen-Huang Cheng, Junmo Kim, Wei-Ta Chu, Peng Cui, Jung-Woo Choi, Min-Chun Hu, and Wesley De Neve

\section{Correction to: \\ Y. M. Ro et al. (Eds.): MultiMedia Modeling, LNCS 11962, https://doi.org/10.1007/978-3-030-37734-2}

The original version of this book was revised. Due to a technical error, the first volume editor did not appear in the volumes of the MMM 2020 proceedings. A funding number was missing in the acknowledgement section of the chapter titled "AttenNet: Deep Attention Based Retinal Disease Classification in OCT Images." Both were corrected.

The updated version of the book can be found at https://doi.org/10.1007/978-3-030-37734-2_46

https://doi.org/10.1007/978-3-030-37734-2 